\title{
A Stiffness Estimator for Agonistic-Antagonistic Variable-Stiffness-Actuator Devices
}

\author{
Tomas Ménard, Giorgio Grioli and Antonio Bicchi
}

\begin{abstract}
Safe Physical Human Robot Interaction, conservation of energy and adaptability are just the main robotic applications that prompted the development of a number of Variable Stiffness Actuators (VSA). Implemented in a variety of ways, they use various technologies, and feature the most diverse mechanical solutions, all of which share a fundamentally unavoidable nonlinear behavior. The control schemes proposed for these actuators typically aim at independent control of the position of the link, and its stiffness. Although effective feedback control schemes using position and force sensors are commonplace in robotics, control of stiffness is at present completely open-loop: the stiffness is inferred from the mathematical model of the actuator. We consider here the problem of estimating the nonlinear stiffness of Variable Stiffness Actuator in Agonistic-Antagonistic configuration. We propose an algorithm based on modulating functions which allow to avoid the need of numerical derivative and for which the tunning is then very simple. An analysis of the error demonstrates the convergence. Simulations are provided and the algorithm is validated on experimental data.
\end{abstract}

\section{INTRODUCTION}

$\mathbf{T}$ HE control of flexibility in actuators is a topic that gained in importance during the last decades, when the possibility to take advantage of the flexibility has become a possible option, rather than minimizing it [24]. Among the resulting applications, one of the most important is safety of physical Human Robot Interaction (phHRI) [1], [2], [15]. In case of accidental collision between a human and a robot, a compliant actuator can reduce the risk of injuries, while the actuator can be made more rigid for precise tasks. Others applications have been developed such as mechanism preservation [11], energy saving [28], energy storing to release it at particular time [8], [12] or rehabilitation [14], [32].

In order to control both position and stiffness, specialized devices have been designed which are made up of two actuators and flexible transmissions presenting nonlinear characteristics [29]. Most existing devices can be classified into two categories, as shown on figure 1. The first approach is bio-inspired and consists in setting the two motors in an Agonistic-Antagonistic configuration, connected to the link via nonlinear spring, similarly to antagonist muscles actuating an arm [20], [27], [22], [3]. In the second approach, called serialconfiguration, the dynamics of the two motors are almost decoupled. A first motor is dedicated to controlling the link motion, while a second smaller motor is dedicated to the setting of the stiffness [4], [16], [30], [17], [13].

After the problem of finding a suitable architecture for VSA, comes the problem of controlling both the position and the stiffness, as illustrated in the example of figure 2. While for the serial configuration the dynamics of the positions and the stiffness are almost decoupled, it is not the case for the Agonistic-Antagonistic configuration, where the dynamics are coupled and nonlinear. Several control laws have hence been proposed in the last decade. For the

Corresponding author: T. Ménard is with GREYC, University of Caen, 6 Bd du Maréchal Juin, 14050 Caen Cedex, France. (e-mail: tomas.menard@unicaen.fr)

G. Grioli and A. Bicchi are with Centro Interdipartimentale di Ricerca "E. Piaggio", University of Pisa, 56126, Italy and Italian Inst. of Technology, Advanced Robotics, 16163 Genova, Italy. (e-mail:bicchi@ing.unipi.it; giorgio.grioli@centropiaggio.unipi.it) regulation of the stiffness and the position to constant values, a feedback control has been proposed in [5] and a port Hamiltonian approach, which allow the regulation while keeping the stored energy constant, in [31]. Control law for tracking smooth trajectory have also been proposed in [6], [23], [33] using a feedback linearization strategy for single DOF and multi DOF actuators and in [26] following a gain scheduling method and LQR for robustness.

All the above control scheme have the same issue, a precise knowledge of the stiffness is required, but there is no sensor for the stiffness. Hence it has to be reconstructed either by a mathematical model, but it is prone to error since the model is usually complex and subject to high imprecision, or with an online estimator. The latter method is far preferable and has been investigated in several papers. A first method has been proposed in [9] where the error estimation was shown to be uniformly ultimately bounded. An improved algorithm has been proposed in [10], where the observer is derived from a parametric approach coupled with an analytic differentiation. Both these methods have been operating on the link side and require several numerical differentiation. In order to alleviate this issue, other algorithms have been developed, working on the motor side. The problem can then be stated as follows, the equation representing the movement of the motor angle $q$ is given by:

$$
\tau=J q^{(2)}+b q^{(1)}-f(\phi)
$$

where $\tau$ is the torque of the motor, $J$ and $b$ are the motor inertia and damping and $\phi=q-q_{L}$ is the displacement angle with $q_{L}$ the link angle, all these parameters and signals are known or measured. The problem is to estimate $\sigma(\phi)=\frac{\partial f}{\partial \phi}$, the stiffness. The main issue is that we need the derivatives of the measured signals which is a difficult problem for noisy signals. To the best of our knowledge, two algorithms have been proposed for the estimation of $\sigma$, following this way.

Firstly, in [7], where the idea is to obtain first an estimation of $f(\phi)$ by combining a modified kinematic Kalman filter for the estimation of the first derivative of $q$ and a first order filter. Then $f$ is approximated by a Taylor expansion and its parameters are estimated by a least square algorithm. Finally, $\sigma$ is obtained by analytically differentiating the Taylor expansion. The main drawback of this method is that the convergence required for this type of algorithm cannot be ensured. These restrictions have been partially alleviated in [21] where an algorithm has been proposed based on operational calculus and differential algebra. The idea is to first obtain a relation involving $\sigma$ by differentiating equation (1), then, by doing some calculus in the Laplace domain, a relation between filtered versions of the measured signals and $\sigma$ is obtained. Replacing $\sigma$ by a Taylor expansion approximation, a Least Square Algorithm can be used, involving filtered versions of the different measured signals in order to estimate the parameters of the Taylor expansion. The fact that no numerical derivative is needed, makes this algorithm very easy to tune, and hence practically interesting, indeed, there are only three parameters to set: the length of the integration window, the initial value of the covariance matrix for the recursive least square algorithm and the order of the Taylor expansion.

We propose here an improved version of [21]. First, the algorithm 


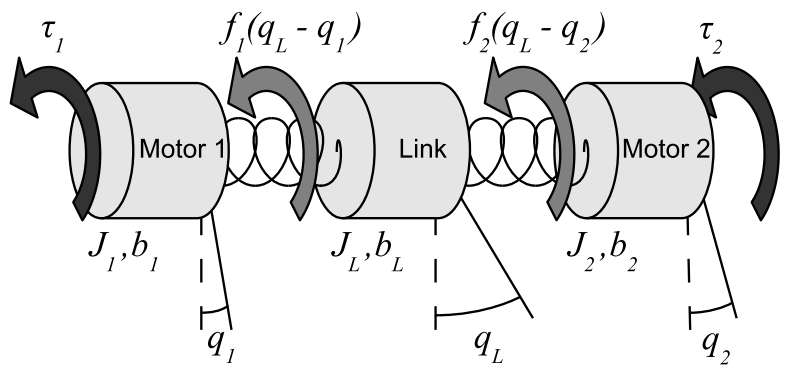

(a) Agonist-Antagonistic VSA

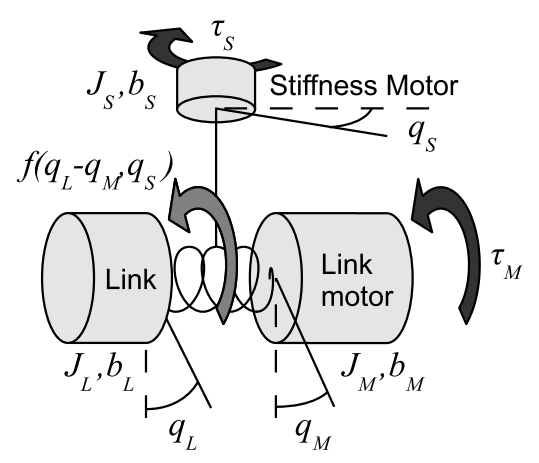

(b) Explicit Stiffness Variator

Fig. 1. Two common categories of Variable Stiffness Actuators.

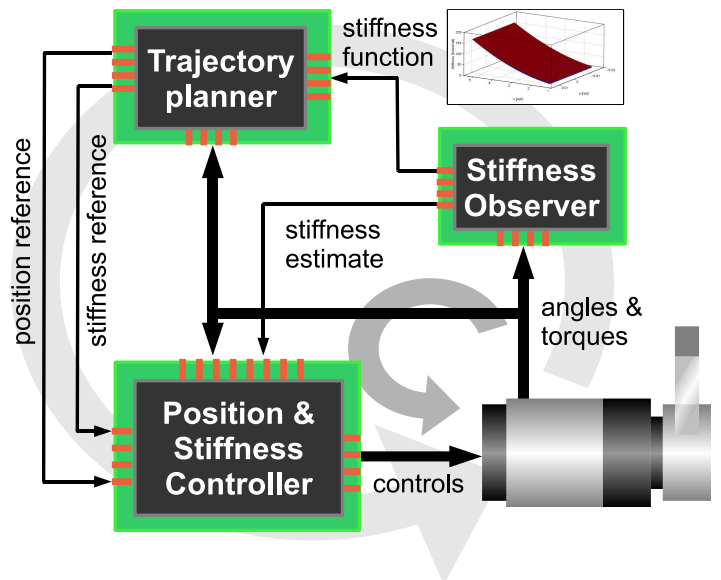

Fig. 2. A possible control system for a Variable Stiffness Actuator, organized in two layers: On the lowest layer a position and stiffness controller tracks the references generated by the higher level trajectory planner. Both control loops rely on knowledge on the stiffness of the actuator: the instantaneous value of the stiffness is used to close the inner loop, while the shape of the stiffness function is used by the trajectory planning algorithm.

is simplified, while in [21] it has been derived using operational calculus and differential algebra, we utilize here modulating functions, which allow to obtain the filters, needed for the algorithm, clearly and easily. In addition, we analyze the effect of the measurement noise and the truncation error on the estimation of the stiffness, and provide a guideline for the tunning of the different parameters and ensure the convergence of the algorithm when there is noise on the torques measurements.

The paper is organized as follow. In section II, we present the class of systems considered in this paper and the definition of stiffness. The main development is done in section III, where the methodology is presented for a VSA in Agonistic-Antagonistic configuration. An analysis of the error on the measured data is given next. The performance of the observer is illustrated on simulations in section IV. The observer is then used on real experimental data, for an AAVSA in section V. Finally, section VI presents our conclusions.

\section{Problem STATEMENT}

We focus our analysis on the Agonistic-Antagonistic VSA devices, which scheme is that of figure 1(a). We recall here the model presented in [27]. We denote $\tau_{1}$ and $\tau_{2}$ the torques of the motors, $f_{1}$ and $f_{2}$ the elastic torques of the variable stiffness, $q_{1}, q_{2}$ and $q_{L}$ the positions of the two motors and the link, respectively. The gravity term is given by $g$. The parameters of the two motors and the link are $J_{1}, J_{2}, J_{L}$ the inertias and $b_{1}, b_{2}, b_{L}$ the dampings, respectively.

The dynamical model is given by:

$$
\begin{aligned}
\tau_{1}= & J_{1} q_{1}^{(2)}+b_{1} q_{1}^{(1)}-f_{1}\left(q_{L}-q_{1}\right) \\
\tau_{2}= & J_{2} q_{2}^{(2)}+b_{2} q_{2}^{(1)}-f_{2}\left(q_{L}-q_{2}\right) \\
\tau_{L}= & J_{L} q_{L}^{(2)}+b_{L} q_{L}^{(1)}+g\left(q_{L}\right) \\
& +f_{1}\left(q_{L}-q_{1}\right)+f_{2}\left(q_{L}-q_{2}\right)
\end{aligned}
$$

and the total flexibility torque is

$$
f(\phi)=f_{1}\left(\phi_{1}\right)+f_{2}\left(\phi_{2}\right) .
$$

where $\phi=\left(\phi_{1}, \phi_{2}\right)$ with $\phi_{i}=q_{L}-q_{i}, i=1,2$, correspond to the deformation of each motor-transmission.

The total device stiffness is derived from the total flexibility torque and is equal to

$$
\sigma(\phi)=\sigma_{1}\left(\phi_{1}\right)+\sigma_{2}\left(\phi_{2}\right)
$$

where

$$
\sigma_{i}\left(\phi_{i}\right)=\frac{\partial f_{i}}{\partial \phi_{i}}\left(\phi_{i}\right), \quad i=1,2
$$

\section{OBSERVER DESIGN FOR STIFFNESS ESTIMATION}

In this section, we first present the modulating functions and give some useful properties. These properties are further used to define the stiffness estimator. Afterward, the effects of the measurement noise and the truncation error are analyzed.

\section{A. Modulating functions}

The following definitions and proposition come from [25], and have been slightly modified for our need.

Definition 1. A modulating function of order $k$ on $[a, b](a, b \in \mathbb{R})$ is a function $\psi:[a, b] \rightarrow \mathbb{R}, k$-times differentiable such that:

$$
\psi^{(i)}(a)=0=\psi^{(i)}(b), \quad i=0, \ldots, k-1,
$$

where $\psi^{(i)}$ represents the $i$-th order derivative of $\psi$.

Definition 2. A function $f:[a, b] \rightarrow \mathbb{R}$ integrable on $[a, b]$ is modulated by taking the inner product with a modulating function $\psi$ :

$$
\langle f, \psi\rangle=\int_{a}^{b} f(u) \psi(u) d u .
$$

Proposition 1. Let $f_{1}, f_{2}$ be integrable real valued functions on $[a, b], \psi$ an order $k$ modulating function on $[a, b]$ and $C \in \mathbb{R} a$ constant, then we have the following properties:

(i) $\left\langle f_{1}^{(i)}, \psi\right\rangle=(-1)^{i}\left\langle f_{1}, \psi^{(i)}\right\rangle, \quad i=0, \ldots, k-1$, 
(ii) $\left\langle C f_{1}+f_{2}, \psi\right\rangle=C\left\langle f_{1}, \psi\right\rangle+\left\langle f_{2}, \psi\right\rangle$.

Property 1 is very important, because it allows to replace the derivative on a function $f$, which is usually unknown (for example, we only have access to a measured signal), by the derivative on the modulating function for which the derivative is known and can be computed analytically.

Example 1. Let us define the following function:

$$
w_{i, j}(u)=(1-u)^{i} u^{j}, \quad u \in \mathbb{R}, i, j \in \mathbb{N} .
$$

Then, $w_{k, k}$, for $k \in \mathbb{N}$, is a modulating function of order $k+1$ on $[0,1]$.

\section{B. Algorithm}

The estimation of the stiffness is derived from equations (2)-(3), that is, we look at the system on the motor side. The algorithm is split into two parts. Firstly, the equations are differentiated to make the stiffness appear explicitly, the stiffness is then approximated by a Taylor expansion and the resulting equations are transformed, using modulating functions, so that only filtered versions of the measured signals are needed. Secondly, a least square algorithm is used to estimate the parameters of the Taylor expansion and thus the stiffness itself. is:

Computation of the filters: We start from equations (2)-(3), that

$$
\tau_{i}=J_{i} q_{i}^{(2)}+b_{i} q_{i}^{(1)}-f_{i}\left(\phi_{i}\right), \quad i=1,2 .
$$

Differentiating with respect to time yields:

$$
\tau_{i}^{(1)}=J_{i} q_{i}^{(3)}+b_{i} q_{i}^{(2)}-\phi_{i}^{(1)} \sigma_{i}\left(\phi_{i}\right),
$$

we take the following Taylor expansion approximation:

$$
\sigma_{i}\left(\phi_{i}\right) \approx \sum_{j=0}^{N} \alpha_{j}^{i} \frac{\left(\phi_{i}\right)^{j}}{j !}
$$

which gives the relation:

$$
\sum_{j=0}^{N} \alpha_{j}^{i} \frac{\phi_{i}^{(1)}\left(\phi_{i}\right)^{j}}{j !}=J_{i} q_{i}^{(3)}+b_{i} q_{i}^{(2)}-\tau_{i}^{(1)} .
$$

The maximum derivative order is 3 , then we need to take an order 4 modulating function $\psi$, which will be defined later. Modulating equation (14) with $\psi$, one obtain:

$$
\begin{aligned}
\left\langle\sum_{j=0}^{N} \alpha_{j}^{i} \frac{\phi_{i}^{(1)}\left(\phi_{i}\right)^{j}}{j !}, \psi\right\rangle= & \left\langle J_{i} q_{i}^{(3)}+b_{i} q_{i}^{(2)}-\tau_{i}^{(1)}, \psi\right\rangle,(15) \\
\sum_{j=0}^{N} \alpha_{j}^{i}\left\langle\left(\frac{\left(\phi_{i}\right)^{j+1}}{(j+1) !}\right)^{(1)}, \psi\right\rangle= & J_{i}\left\langle q_{i}^{(3)}, \psi\right\rangle+b_{i}\left\langle q_{i}^{(2)}, \psi\right\rangle \\
& -\left\langle\tau_{i}^{(1)}, \psi\right\rangle \\
\sum_{j=0}^{N} \alpha_{j}^{i}\left\langle\frac{\left(\phi_{i}\right)^{j+1}}{(j+1) !}, \psi^{(1)}\right\rangle= & J_{i}\left\langle q_{i}, \psi^{(3)}\right\rangle-b_{i}\left\langle q_{i}, \psi^{(2)}\right\rangle(17) \\
& -\left\langle\tau_{i}, \psi^{(1)}\right\rangle .
\end{aligned}
$$

We get a relation between the stiffness and the measured signals, where the only source of error is the Taylor approximation. We want to estimate the parameters $\alpha_{i}$ with a least square algorithm, then we need a relation changing with time $t$. For this purpose, we take $a=$ $t-T$ and $b=t$, where $T>0$ is the length of the integration window and the modulating function is taken as $\psi(u)=(u-t+T)^{3}(t-u)^{3}$. We have the following relation:

$$
\sum_{j=0}^{N} \alpha_{j}^{i} \int_{t-T}^{t} \frac{\left(\phi_{i}\right)^{j+1}}{(j+1) !}(u) \psi^{(1)}(u) d u=J_{i} \int_{t-T}^{t} q_{i}(u) \psi^{(3)}(u) d u
$$

$$
\begin{gathered}
-b_{i} \int_{t-T}^{t} q_{i}(u) \psi^{(2)}(u) d u \\
-\int_{t-T}^{t} \tau_{i}(u) \psi^{(1)}(u) d u .
\end{gathered}
$$

Doing the change of variables $u=T \nu+t-T$ and dividing by $T^{3}$ yields

$$
\begin{aligned}
\sum_{j=0}^{N} \alpha_{j}^{i}\left(T^{2} \int_{0}^{1} \frac{\left(\phi_{i}\right)^{j+1}}{(j+1) !}(t+T(\nu-1))\right. & \left.w_{3,3}^{(1)}(\nu) d \nu\right)= \\
& +J_{i} \int_{0}^{1} q_{i}(t+T(\nu-1)) w_{3,3}^{(3)}(\nu) d \nu \\
& -b_{i} T \int_{0}^{1} q_{i}(t+T(\nu-1)) w_{3,3}^{(2)}(\nu) d \nu \\
& -T^{2} \int_{0}^{1} \tau_{i}(t+T(\nu-1)) w_{3,3}^{(1)}(\nu) d \nu,
\end{aligned}
$$

the function $w_{3,3}$ is defined by equation (10). The last thing to do is to obtain a discrete version of this relation. We assume that the sampling period is $T_{s}$ and that $T=M T_{s}$, where $M \in \mathbb{N}$. We take an approximation of the integral with the trapezoidal method, that is:

$$
\int_{0}^{1} f(u) d u \approx \sum_{m=0}^{M} W_{m} f\left(t_{m}\right),
$$

with $t_{m}=m T_{s}, W_{0}=W_{M}=T_{s} / 2$ and $W_{m}=T_{s}, m=$ $1, \ldots, M-1$.

We finally obtain the following relation, at discrete-time $k$ (corresponding to the continuous time $t=k T_{s}$ ):

$$
\begin{aligned}
C_{i}(k) & =\sum_{j=0}^{N} \alpha_{j}^{i} b_{j}^{i}(k), \\
& \triangleq A_{i}^{T} B_{i}(k),
\end{aligned}
$$

with $A_{i}=\left[\alpha_{0}^{i}, \ldots, \alpha_{N}^{i}\right]^{T}, B_{i}(k)=\left[b_{0}^{i}, \ldots, b_{N}^{i}\right]^{T}$,

$$
C_{i}(k)=\sum_{m=0}^{M} q_{i}\left((k-m) T_{s}\right) \times
$$

$$
\begin{array}{r}
\left.\left(J_{i} W_{m} w_{3,3}^{(3)}\right)(m / M)-b_{i} W_{m} T w_{3,3}^{(2)}(m / M)\right) \\
+\sum_{m=0}^{M} \tau_{i}\left((k-m) T_{s}\right)\left(-T^{2} W_{m} w_{3,3}^{(1)}(m / M)\right),
\end{array}
$$

and

$$
b_{j}^{i}(k)=\sum_{m=0}^{M}\left(\frac{\phi_{i}^{j+1}}{(j+1) !}\right)\left((k-m) T_{s}\right)\left(T^{2} W_{m} w_{3,3}^{(1)}(m / M)\right) .
$$

For $k \leq M$, measured values at negative time $(k-m) T_{s}$, i.e. $(k-$ $m)<0$, are set to zero. The derivatives of $w_{3,3}$ are given by:

$$
\begin{aligned}
& w_{3,3}^{(1)}(u)=-3 w_{2,3}+3 w_{3,2}, \\
& w_{3,3}^{(2)}(u)=6 w_{1,3}(u)-18 w_{2,2}(u)+6 w_{3,1}(u), \\
& w_{3,3}^{(3)}(u)=-6 w_{0,3}(u)+54 w_{1,2}(u)-54 w_{2,1}(u)+6 w_{3,0}(u) .
\end{aligned}
$$

Note that the definition of $B_{i}$ and $C_{i}$ can be seen as filtering by a Finite Impulse Response Digital Filter.

Recursive least square algorithm: Now that a relation has been obtained between the stiffness parameters in the Taylor expansion and the measured signals, we further use it into a discrete least square algorithm in order to obtain an approximation $\hat{A}_{i}$ of $A_{i}$.

$$
\left\{\begin{array}{l}
\hat{A}_{i}(k)=\hat{A}_{i}(k-1)+\Gamma(k) \tilde{C}_{i}(k / k-1), \\
\tilde{C}_{i}(k / k-1)=C_{i}(k)-B_{i}(k) \hat{A}_{i}(k-1), \\
\Gamma(k)=\frac{F(k-1) B_{i}(k)}{1+B_{i}^{T}(k) F(k-1) B_{i}(k)} \\
F(k)=\left(I_{N+1}-\Gamma(k) B_{i}(k)^{T}\right) F(k-1) .
\end{array}\right.
$$

The notation $I_{N+1}$ stands for the identity matrix of dimension $N+1$. 


\begin{tabular}{|c|c|c|c|c|c|}
\hline & $\begin{array}{c}N=2 \\
\text { no noise }\end{array}$ & $\begin{array}{c}N=2 \\
\text { noise on torques }\end{array}$ & $\begin{array}{c}N=2 \\
\text { noise on torques } \\
\text { and positions }\end{array}$ & $\begin{array}{c}N=4 \\
\text { noise on torques }\end{array}$ & $\begin{array}{c}N=4 \\
\text { noise on torques } \\
\text { and positions }\end{array}$ \\
\hline \hline MSE & $3.410^{-2}$ & 19.3 & 38.4 & 39.9 & 56.7 \\
\hline \hline MSREP & $510^{-5}$ & $4.810^{-2}$ & $110^{-1}$ & $8.710^{-2}$ & $1.210^{-1}$ \\
\hline
\end{tabular}

TABLE I

EVALUATION OF SIMULATION RESULTS.

\section{Error analysis}

The algorithm presented here is made up of two parts, first, a relation between filtered versions of the measured data and the coefficients of the Taylor expansion of the stiffness is derived. It is then used in a least square algorithm. In this scheme, there are three sources of errors: a noise on the measurements, an error due to the truncation of the Taylor expansion and an error due to the numerical integration. The noise on the measured data is assumed to be an additional stochastic white noise with zero mean and finite variance and the numerical integration error is assumed to be negligible. The attenuation of the error is only done by the first step, that is we will see how to set the parameters to reduce sufficiently the noise so that it does not affect the second step.

We shall treat here measurements noise only for the torque. High noise on the positions measurements would be a critical issue for the algorithm presented here, but, since sensors for positions are far more accurate than for torque measurement, assuming small noise on the positions measurements is a realistic assumption. Furthermore, the effect of a small noise on the positions will be studied on simulations only.

Note that the input of the RLS at time $t$ is a filtered version of the measured signals and then depend on the evolution of the measured signals between $t-T$ and $t$, but since the parameters to be estimated are assumed to be constant, it does not induce further problems. This could, however, be the case for non constant parameters.

Taking errors into account, equation (21), can be rewritten as follows:

$$
A_{i}^{T} B_{i}(k)=C_{i}(k)+e_{R_{N}}^{i}(k)+e_{\omega}^{i}(k),
$$

where $e_{R_{n}}(k)$ is the error due to the truncation and $e_{\omega}(k)$ is the error due to the measurement noise. Three different parameters can be used to reduce the error: the length of the integration window $T$, the order of the Taylor expansion $N$ and the sampling period $T_{s}$.

Analysis of $e_{R_{N}}$

We start from equation (14), replacing the approximation of the stiffness by its true expression and following the same computations as for the algorithm, we obtain:

$$
e_{R_{N}}^{i}(k)=\sum_{m=0}^{M}\left(\sigma_{i}-\sigma_{i}^{N}\right) \circ\left(\phi_{i}\right)\left((k-m) T_{s}\right) T^{2} W_{m} w_{3,3}^{(1)}(m / M),
$$

where $\sigma_{i}^{N}$ is the Taylor expansion of $\sigma_{i}$ up to order $N$. We see, from the expression of the truncation error, that in order to get a bound, the transmission deformation $\phi_{i}$ has to be bounded. Hence, we assume that there exist $\varepsilon_{1}^{i}, \varepsilon_{2}^{i}$ such that $\phi_{i}(k) \in\left[\varepsilon_{1}^{i}, \varepsilon_{2}^{i}\right]$ for all $k \geq 0$, then, overvaluating $\left(\sigma_{i}-\sigma_{i}^{N}\right) \circ\left(\phi_{i}\right)$ and applying Proposition 2, one obtains

$$
\left|e_{R_{N}}^{i}(k)\right| \leq T^{3} \frac{3 \sqrt{5}}{125} \sup _{\phi_{i} \in\left[\varepsilon_{1}^{i}, \varepsilon_{2}^{i}\right]}\left|\sigma_{i}\left(\phi_{i}\right)-\sigma_{i}^{N}\left(\phi_{i}\right)\right| .
$$

Analysis of $e_{\omega}$

We assume here that $\omega$ is a white noise with zero mean and finite variance. Similarly to the truncation error, we obtain that the noise error contribution is equal to

$$
e_{\omega}(k)=\sum_{m=0}^{M} \omega\left((k-m) T_{s}\right) T^{2} W_{m} w_{3,3}^{(1)}(m / M) .
$$

Note that the error due to the noise on the torques does not depend on the subscript $i$ (the error is the same for both torques).

Applying proposition 3 , we obtain that for each $k \in \mathbb{N}, e_{\omega}(k)$ converges to zero in mean square as $M$ goes to infinity.

\section{Parameters settings}

The effect of the parameters on the different errors is summarized in the table II. From the previous analysis, we can derive some indications for the tuning of the parameters. First, the sampling period $T_{s}$ should be taken, as small as possible in order to reduce the effect of the noise. The length of the integration window should be taken large enough to filter the noise, depending on the expected Signal to Noise Ratio which itself depends on the sensors. Even if increasing $T$ will increase the truncation error, we can see from equation (30), that this relation is cubic, and since, typically, values of $T$ belong to $[0.1,2]$, the setting of $T$ and $N$ can be done independently. Finally, the value of $N$ will highly depend on the range of the motor/link deformations $\phi_{i}$ and the spring.

\section{Convergence of the RLS}

We have shown until now that the error contribution can be made arbitrarily small, uniformly with respect to time, by tuning the parameters. According to lemma 1, if the input of the RLS is persistently exciting, then a small error due to the truncation of the Taylor expansion leads to a small error on the estimated coefficients. Similarly, if the error due to the noise converges to zero in mean square, then the estimated parameters will converge in mean square toward the real one.

We thus have to be sure that the persistent excitation condition is fulfilled. The inputs of the RLS are filtered versions of the measured signals. Basically, this filter corresponds to integrating the measured signal over a finite horizon window, and then acts as a low pass filter whose cut-of frequency is proportional to $1 / T$ [25]. We can see from this analysis that if the movements of the link were too fast compared to the cut-of frequency, then, the persistent excitation could be compromised. In this case, it would be necessary to reduce the length $T$ of the integration window. If we want to continue filtering the error due to the noise we need to take a $T_{s}$ smaller (which is natural if the movements become faster).

Finally, we conclude that we can ensure arbitrary small error on the stiffness by tunning the algorithms parameters.

TABLE II

EFFECT OF PARAMETERS ON THE DIFFERENT TYPES OF ERROR..

\begin{tabular}{|c|c|c|}
\hline & Truncated term error & Noise error contribution \\
\hline$N \uparrow$ & $\searrow$ & $\longrightarrow$ \\
\hline$T_{s} \uparrow$ & $\longrightarrow$ & $\searrow$ \\
\hline$T \uparrow$ & $\nearrow$ & $\searrow$ \\
\hline
\end{tabular}




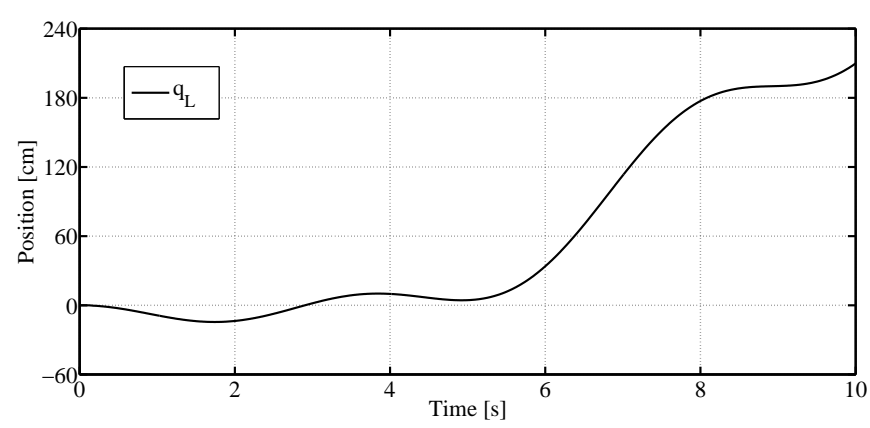

(a) position of the link

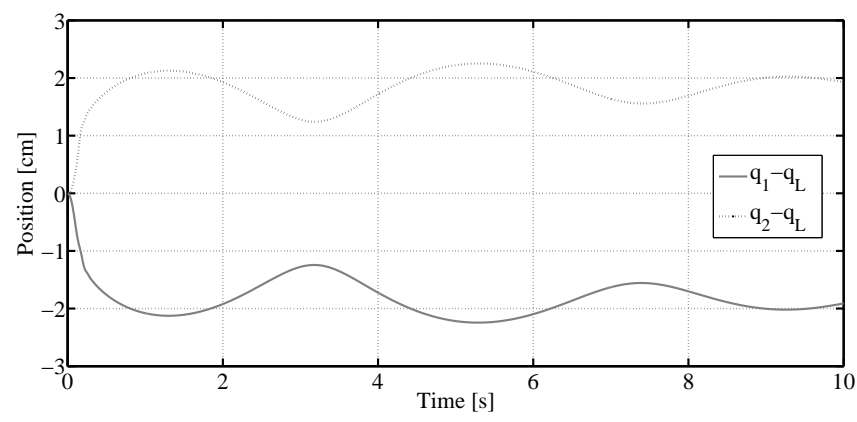

(b) displacements

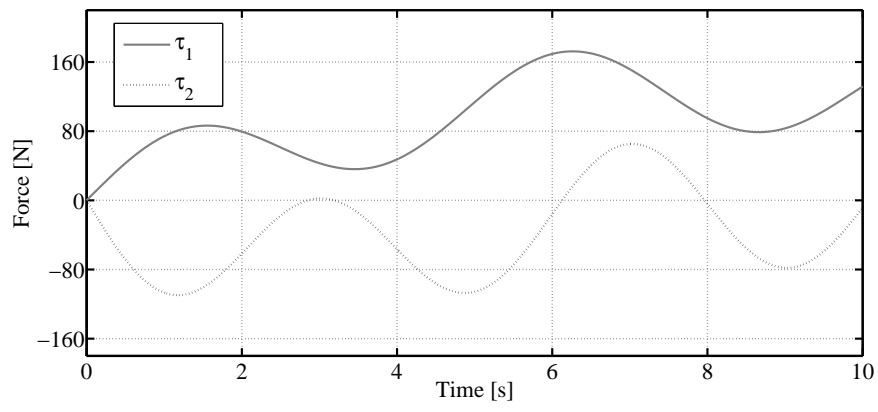

(c) torques

Fig. 3. Data used for the simulations Agonistic-Antagonistic VSA.

\section{Simulations}

We consider an Agonistic-Antagonistic VSA mechanism realized with two identical cubic springs whose force displacement characteristic are described by:

$$
f_{i}=10\left(q_{L}-q_{i}\right)^{3}, i=1,2 .
$$

The parameters of the motors in the equations (2)-(3)-(4) are given by, $J_{1}=J_{2}=10^{-4} \mathrm{~kg} / \mathrm{m}^{2}, J_{L}=0.0179 \mathrm{~kg} / \mathrm{m}^{2}, b_{1}=b_{2}=$ $1.27 \mathrm{Nms} / \mathrm{rad}$ and $b_{L}=0.0127 \mathrm{Nms} / \mathrm{rad}$. We consider the case without gravity, that is $g=0$.

We provide in this section the result of several simulations with the algorithm proposed here, all the simulations are done for the same set of data, which are reported on figure 3 .

In order to precisely evaluate the effects of noise on the reconstruction of the stiffness, three different cases are compared in simulation: without noise, with a white noise affecting the torques and finally with a white noise affecting both the torques and the positions. The importance of the noise is quantified by the Signal to Noise Ratio (SNR) which is given by the formula $S N R=$ $20 \log _{10}(\operatorname{Var}($ Signal without noise $) / \operatorname{Var}($ Noise $))$, the lower it

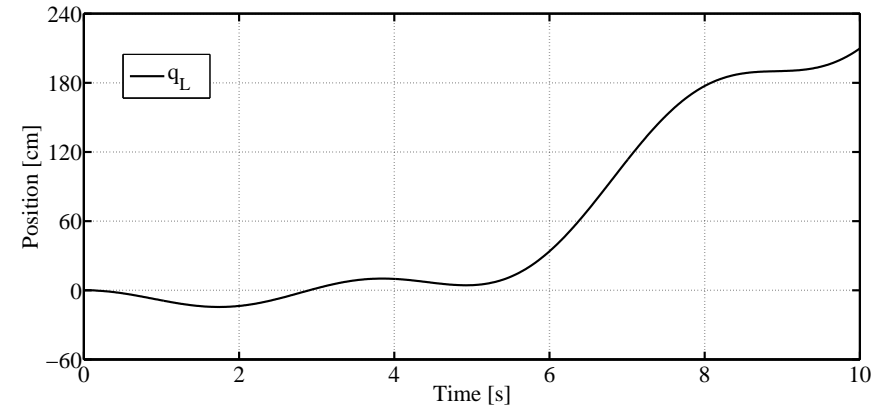

(a) position of the link

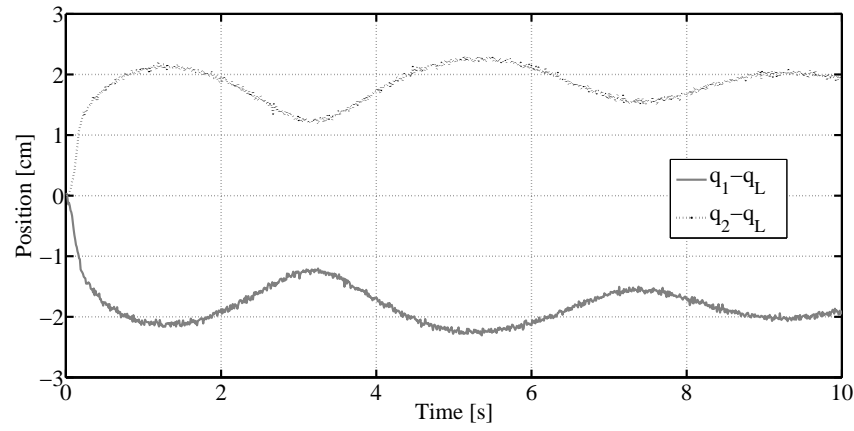

(b) displacements

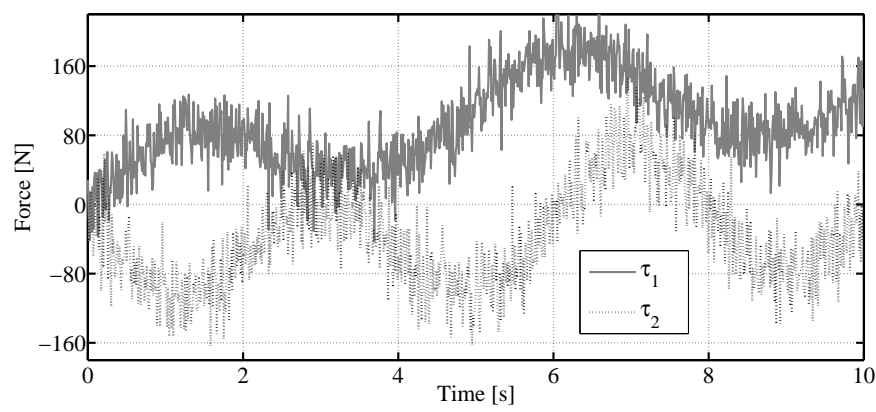

(c) torques

Fig. 4. Noisy signals used for the simulations.

is, the more significant the noise is. The noise on the torques is taken large, corresponding to a SNR of 9, while the noise on the positions is low corresponding to a SNR of 140 , noisy signals are depicted on figure 4. In addition, we compare the effect of $N$ in equation (13), for the estimation. The best value for $N$ is 2 for our algorithm, because the springs are cubics. But, since the springs might be not polynomials (we can consider exponential springs for example), we then test our algorithm for non exact values of $N$, that is, with $N=2$ and $N=4$.

\section{set-up for the algorithm}

The parameters of the observer are set as follow, the length of the time window for the integral is $T=0.5 s$ and the covariance matrix for the RLS is initialized at $F(0)=10^{8} I_{N+1}$. The sampling time is taken as $T_{s}=1 / 1000 \mathrm{~s}$.

\section{results of simulations}

The results of the reconstruction of the stiffness are given on figure 5. In addition, an average of the Mean Square Error (MSE) and the Mean Square Relative Error Percentage (MSREP) have been computed, over 100 simulations, after the algorithm has converged, that is between $t=2 s$ and $t=10 \mathrm{~s}$ and are given in table I. The formula for MSE and MSREP between sample $k_{1}$ and $k_{2}\left(k_{2}>k_{1}\right)$, 


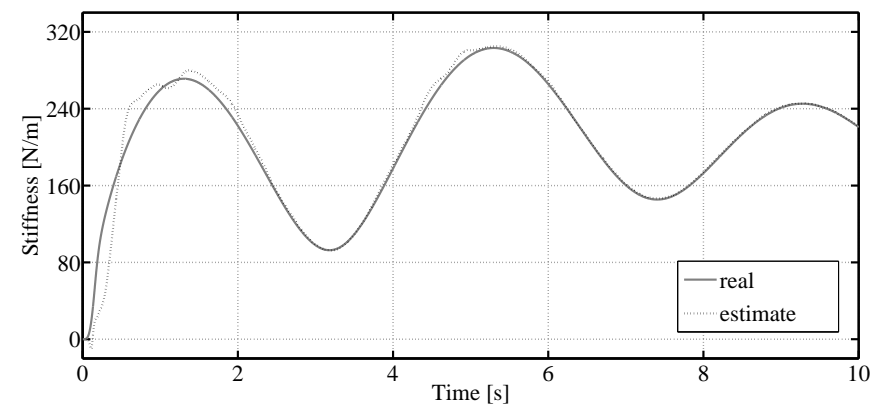

(a) Noisy torques, clear positions, $N=2$

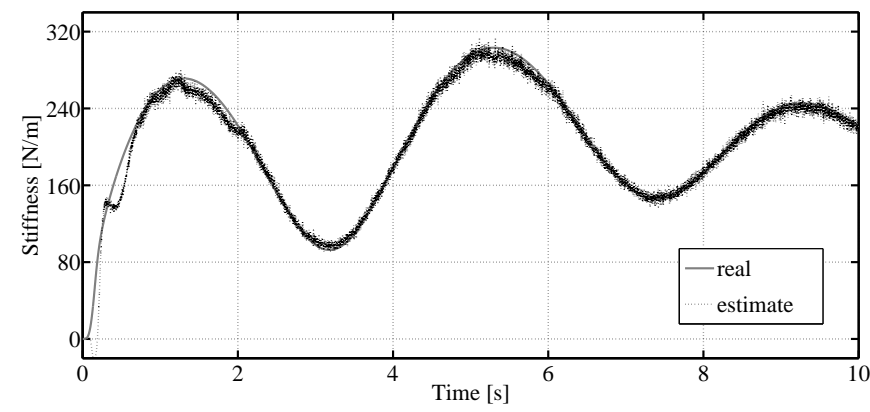

(b) Noisy torques and positions, $N=2$

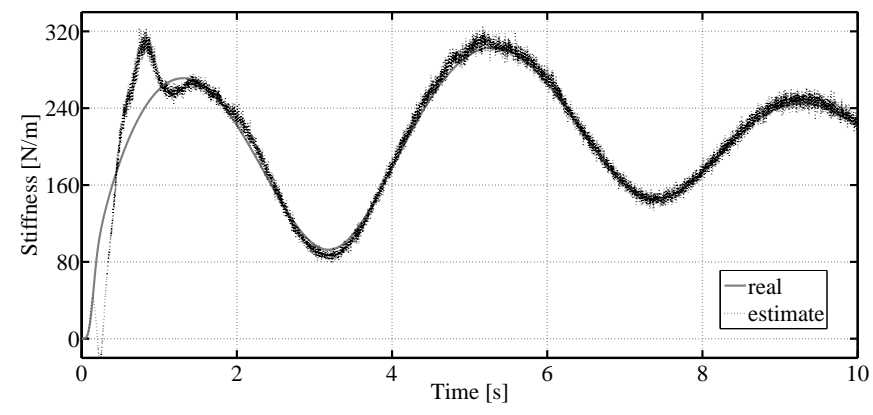

(c) Noisy torques and positions, $N=4$

Fig. 5. Reconstruction of the stiffness in simulation.

are given by:

$$
\begin{aligned}
M S E & =\frac{1}{k_{2}-k_{1}+1} \sum_{k=k_{1}}^{k_{2}}(\sigma(k)-\hat{\sigma(k)})^{2} \\
M S R E P & =\frac{1}{k_{2}-k_{1}+1} \sum_{k=k_{1}}^{k_{2}}\left(\frac{\sigma(k)-\sigma \hat{(k)}}{\sigma(k)}\right)^{2} .
\end{aligned}
$$

We can see, that in every cases and with the same settings, the algorithm performs satisfactorily.

\section{EXPERIMENTAL RESULTS}

In this section, we test the algorithm presented here on the Agonistic-Antagonistic VSA experimental device with exponential springs shown in figure 6, which is fully described in [10] (some details are also reported in appendix for convenience). The system measures are, by nature, translational (see appendix for detail) and the model can be described with the model in figure 7. Please note that this choice of translational Lagrangian coordinates, yields that displacements are measured in $\mathrm{mm}$, generalized torques in $N$ and stiffness in $N / m m$.

The results of the estimations are depicted on figure 9. The comparison model is not exact due to uncertainties in the model

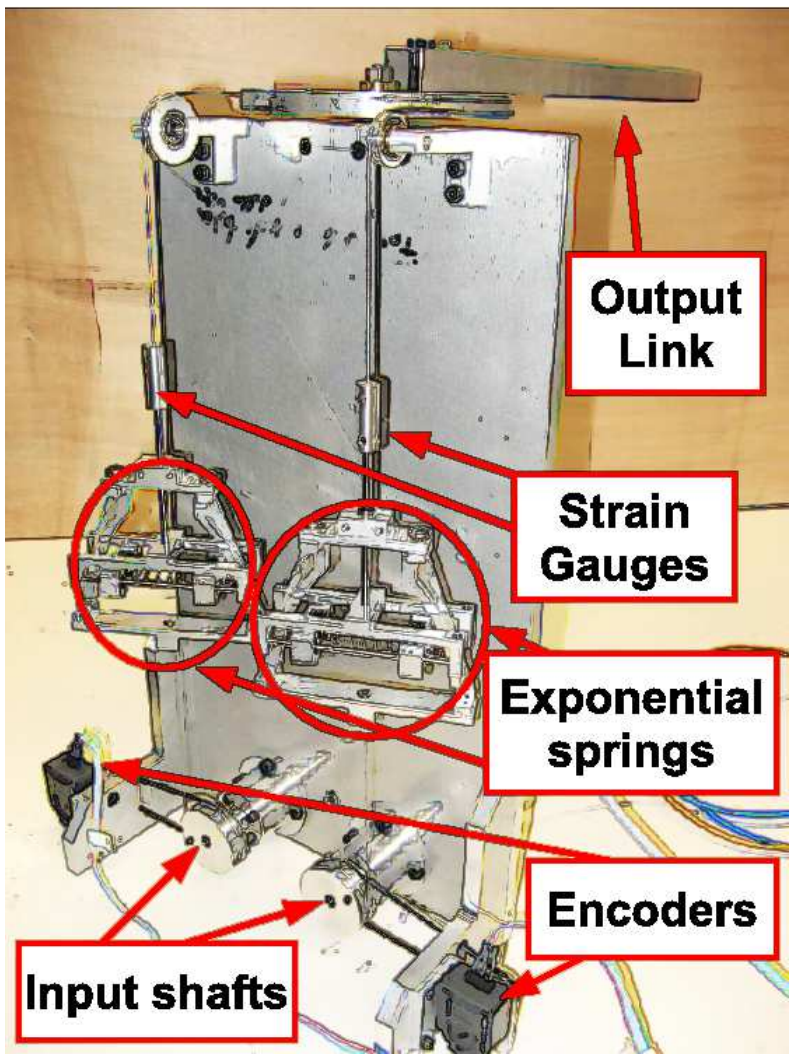

Fig. 6. The experimental setup consists of an Agonistic-Antagonistic VSA system with exponential springs, realized using a linear spring forced to move on a suitable cam profile. Force sensors (strain gauges) are mounted on the tendons connecting the springs to the link. Position sensors (encoders) are mounted on the link and on two tendon pulleys coupled with the input levers.

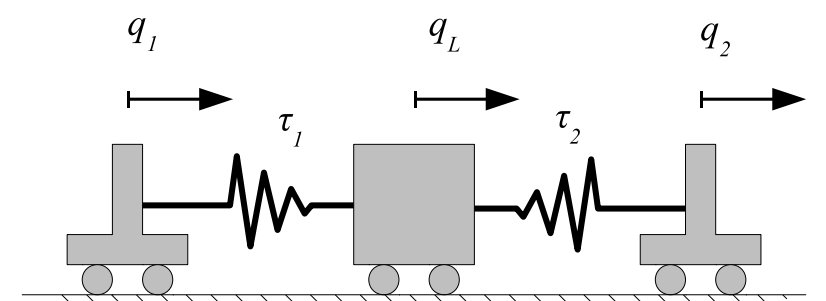

Fig. 7. Translational model of a VSA, as the one used in the experimental setup.

of the actuator and especially in the model parameters. Then, we consider that the knowledge of the true stiffness is reliable up to an error about $25 \%$ (see appendix, in particular figure 10 for details), represented by an horizontal line on figure 9.b).

The order of the Taylor expansion has been set to $N=9$. The covariance matrix for the RLS has been set to $F(0)=10^{5} I_{10}$ and the length of the time window for the integral is $T=0.5 \mathrm{~s}$. The MSE and MSREP, after the convergence (that is after $t=2 s$ ) are equal to 21.43 and 2.07 respectively.

\section{CONCLUSION}

In this paper, we have presented a new algorithm for estimating the nonlinear stiffness of Agonistic-Antagonistic Variable Stiffness Actuator. The advantages of the proposed method are twofold, firstly, the tuning is simple, secondly, the algorithm is proven to 


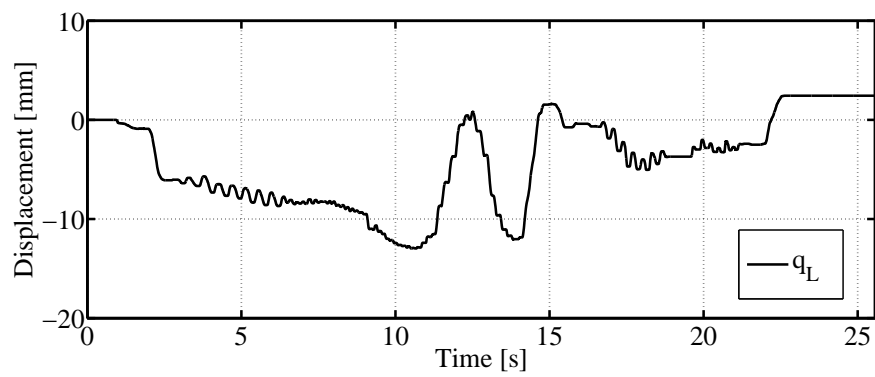

(a) position of the link

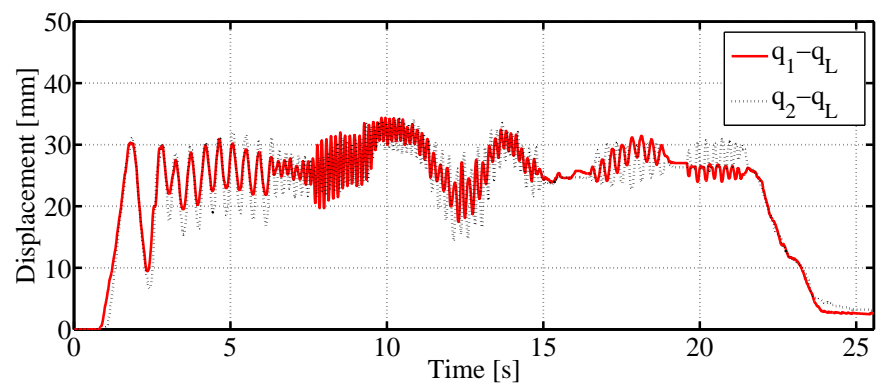

(b) displacements

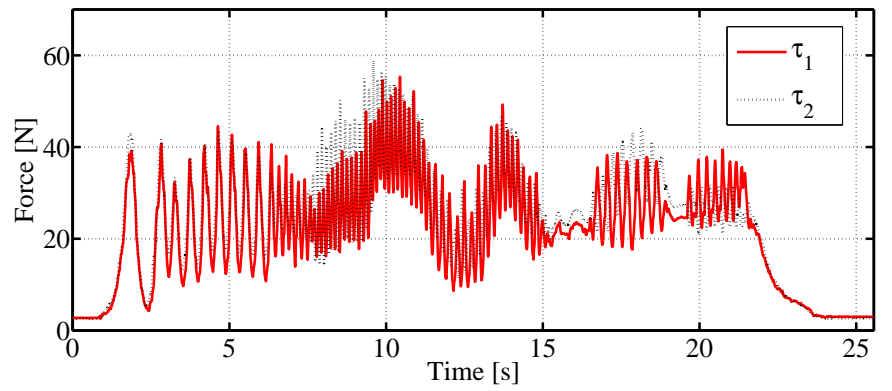

(c) torques

Fig. 8. Experimental data: position, displacement and torque data.

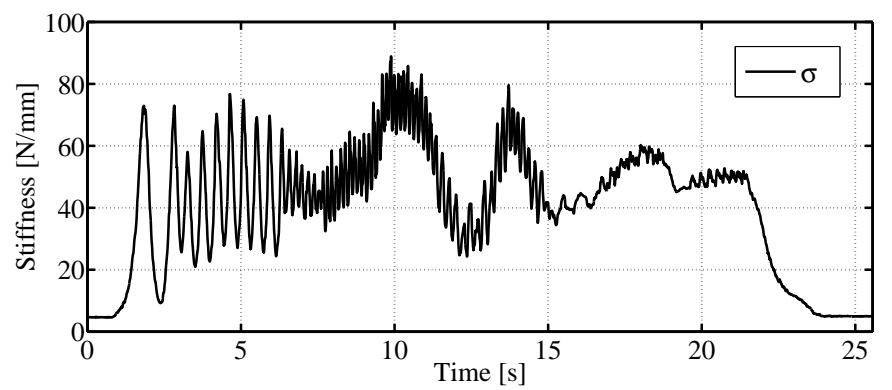

(a) total stiffness

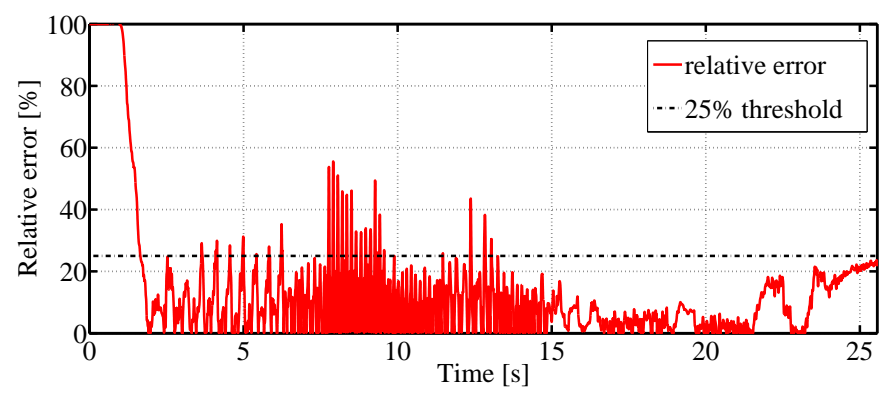

(b) relative error

Fig. 9. Results of experiment: performance of the observer tracking the stiffness of an Agonist-Antagonist VSA. converge. The effect of the noise on the resulting estimation has been studied and a methodology to tune the parameters has been provided. Furthermore, the algorithm has been validated on experimental data which prove its practical efficiency. The proposed algorithm assumes a good knowledge of the damping and inertia on the link side for proper functioning: while a good knowledge of the inertial details of a robot is something that can be easily extracted from the combined information of components datasheets, CAD files and kinematics of the robot, a good knowledge of the damping coefficient is not always easily available. Moreover, damping is often a time-varying quantity, dependent on the age of the system, the temperature and other issues. This aspect opens a really interesting research direction, that is the combined estimation of stiffness and damping, which is demanded to future work.

\section{APPENDIX}

\section{A. Technical results}

The two propositions given here can be seen as specific cases (but slightly different) of Proposition 2 and corollary 2 in [18]. The lemma presents a classical result for RLS and is presented with more details in [19].

Proposition 2. Let $M \in \mathbb{N}$, and $T>0$ then the following inequality holds true

$$
\sum_{m=0}^{M} W_{m} w_{3,3}^{(1)}\left(\frac{m}{M}\right) \leq T \frac{3 \sqrt{5}}{125}
$$

where $W_{0}=W_{M}=T /(2 M), W_{m}=T / M, m=1, \ldots, M-1$ and $w_{3,3}^{(1)}$ is given by (24).

Proof of proposition 2. One has:

$$
\begin{aligned}
\sum_{m=0}^{M} W_{m} w_{3,3}^{(1)}\left(\frac{m}{M}\right) & \leq \max _{u \in[0,1]}\left|w_{3,3}^{(1)}(u)\right| \sum_{m=0}^{M} W_{m}, \\
& \leq \max _{u \in[0,1]}\left|w_{3,3}^{(1)}(u)\right| T, \\
& \leq T \frac{3 \sqrt{5}}{125} .
\end{aligned}
$$

The third inequality is obtained by noticing that the function $w_{3,3}^{(1)}(u)$ is a polynomial function and vanish at $u=0$ and $u=1$. Then the maximum of $\left|w_{3,3}^{(1)}(u)\right|$ is attained at a point, belonging to $[0,1]$, where the derivative of $w_{3,3}^{(1)}(u)$ vanish. Direct computations gives that $w_{3,3}^{(2)}(u)$ vanish at $0, \frac{1}{2}+\frac{\sqrt{5}}{10}, \frac{1}{2}-\frac{\sqrt{5}}{10}, 1$. Hence one can directly conclude that the maximum is equal to $\frac{3 \sqrt{5}}{125}$.

Proposition 3. Consider $\{\omega(t), t>0\}$, a continuous parameter stochastic process, such that for every $t>0$ :

$$
\begin{aligned}
E[\omega(t)] & =0, \\
\operatorname{Cov}[\omega(t) \omega(s)] & =0, \quad t \neq s, \\
\operatorname{Var}[\omega(t)] & =\mu^{2} .
\end{aligned}
$$

Let $M \in \mathbb{N}$, and $T>0$, then, for every $k \in \mathbb{N}, k \geq M$ :

$$
\lim _{M \rightarrow+\infty} e_{\omega}(k) \stackrel{\text { m.s. }}{=} 0
$$

where m.s. stands for mean square convergence, and

$$
e_{\omega}(k)=\sum_{m=0}^{M} W_{m} \omega\left((k-m) \frac{T}{M}\right) w_{3,3}\left(\frac{m}{M}\right),
$$

where $W_{0}=W_{M}=T /(2 M), W_{m}=T / M, m=1, \ldots, M-1$ and $w_{3,3}^{(1)}$ is given by (24). 
Proof of proposition 3. Mean square convergence to zero for a stochastic process means that its second-order moment converges towards zero. That is, we have to prove here that $\lim _{M \rightarrow+\infty} E\left[\left(e_{\omega}^{i}(k)\right)^{2}\right]=0$. For this purpose, we will use the wellknown formula:

$$
E\left[\left(e_{\omega}(k)\right)^{2}\right]=\operatorname{Var}\left[e_{\omega}(k)\right]+\left(E\left[e_{\omega}(k)\right]\right)^{2} .
$$

One has:

$$
E\left[e_{\omega}(k)\right]=T^{2} \sum_{m=0}^{M} W_{m} w_{3,3}^{(1)}\left(\frac{m}{M}\right) E\left[\omega\left((k-m) T_{s}\right)\right],
$$

and:

$$
\begin{array}{r}
\operatorname{Var}\left[e_{\omega}(k)\right]=T^{4} \sum_{m=0}^{M} W_{m}^{2}\left(w_{3,3}\left(\frac{m}{M}\right)\right)^{2} \operatorname{Var}\left[\omega\left((k-m) T_{s}\right)\right] \\
+2 \sum_{m_{1}=0}^{M} \sum_{m_{2}=m_{1}+1}^{M} W_{m_{1}} W_{m_{2}} w_{3,3}^{(1)}\left(\frac{m_{1}}{M}\right) w_{3,3}^{(1)}\left(\frac{m_{2}}{M}\right) \\
\operatorname{Cov}\left[\omega\left(\left(k-m_{1}\right) T_{s}\right) \omega\left(\left(k-m_{2}\right) T_{s}\right)\right] .
\end{array}
$$

The hypothesis give:

$$
\begin{aligned}
E\left[\omega\left((k-m) T_{s}\right)\right] & =0, \\
\operatorname{Cov}\left[\omega\left(\left(k-m_{1}\right) T_{s}\right) \omega\left(\left(k-m_{2}\right) T_{s}\right)\right] & =0, \quad \text { if } m_{1} \neq m_{2} .
\end{aligned}
$$

It follows that:

$$
\begin{aligned}
E\left[e_{\omega}(k)\right] & =0 \\
\operatorname{Var}\left[e_{\omega}(k)\right] & =T^{4} \sum_{m=0}^{M} W_{m}^{2}\left(w_{3,3}^{(1)}\left(\frac{m}{M}\right)\right)^{2} \operatorname{Var}\left[\omega\left((k-m) T_{s}\right)\right] \\
& =\frac{T^{4}}{M^{2}} \sum_{m=0}^{M}\left(M W_{m}\right)^{2}\left(w_{3,3}^{(1)}\left(\frac{m}{M}\right)\right)^{2} \times \\
& \leq \frac{T^{4}}{M^{2}} \max _{u \in[0,1]}\left|w_{3,3}^{(1)}\right|^{2} T^{2} \mu^{2} M \\
& \leq \frac{T^{6} \max _{u \in[0,1]}\left|w_{3,3}^{(1)}\right|^{2} \mu^{2}}{M}
\end{aligned}
$$

The last term goes to zero when $M$ goes to infinity since $T$ is fixed and $w_{3,3}^{(1)}$ is a continuous function on $[0,1]$. This ends the proof.

Lemma 1 ([19] p.177). If the vector $B_{i}$ is persistently excited, then the solution of the RLS algorithm (27) is given by:

$$
\hat{A}_{i}(k)=A_{i}+\left[R_{i}(k)\right]^{-1} \frac{1}{k} \sum_{j=1}^{k} B_{i}(j)\left(e_{R_{N}}^{i}(j)+e_{\omega}^{i}(j)\right),
$$

with

$$
R_{i}(k)=\frac{1}{k} \sum_{j=1}^{k} B_{i}(j) B_{i}(j)^{T}
$$

\section{B. Details on the Experimental Setup}

This appendix briefly recalls some details of the experimental setup shown in figure 6, and thoroughly described in [9].

The experimental device implements the antagonistic VSA concept, 1(a). The springs used in the device are designed (using a cam mechanism) to have an exponential characteristic, such that, given the spring deformation $\delta$ and the spring force $f$, the relationship between the two can be summarised as

$$
f=a_{i} e^{b_{i} \delta}
$$

where the subscript $i$ can be 1 or 2 , for the two springs.

A pair of strain-gauge load cells measures the tendon tensions. The three position corresponding to the link position and to the two movement sources were measured using three HEDS-5540 encoders with a resolution of 2000 CPRs. A National Instruments PCI6251 ADC board acuired the strain gauges data, and an USB-PCI4e acquired the encoders data, both with a sampling rate $T_{s}=0.015 \mathrm{~s}$. A second-order filter with time constant of $0.02 s$ was used to clear the signal from the (rather small) noise. Sources of noise were quantization for the encoders and electro-magnetic noise interferences in the strain gauges.

It is important to notice that, although the device is designed as the actuator of a rotary link, part of the measurements acquired are in the rotary domain (the link and prime movers position), while the other part is in the translational domain (the spring forces). To apply the proposed estimation algorithm, all measurements need to be reported in the same domain. The authors of [9] chose to adopt the translational domain, and we kept the same decision to allow easier comparison between the results. This leads to all results presented in figures 8,9 , and 10, that expressed in terms of displacements $(\mathrm{mm})$ and forces $(N)$, rather than angles $(\mathrm{rad})$ and torques $(\mathrm{Nm})$. This does not hinder the validity of reported results in any way, given the substantial equivalence of the rotational and translational domains when limited a system as simple as that used.

To keep the system simple, the motion of the two prime movers was input directly by hand, with the aid of two handles (not shown in figure 6).

To compare the estimation of the stiffness derived from the proposed method with the ground-truth data, the force functions of the two springs need to be experimentally evaluated through careful preliminary calibration experiments. The calibration procedure consists in the collection of a large number of force-displacement pairs $(x, f)$ and then in a subsequent fitting of the pairs with the model 38.

Figure 10, resumes the results of such calibration procedure. It is possible to notice how, notwithstanding the fact that the springs were explicitly designed to show an exponential characteristic, the ground-truth data shows some deviation with respect to the intended model, yielding a relative error (during the regression) of roughly $25 \%$ (precisely a relative error of $22 \%$ and $29 \%$ for the left and right spring, respectively). This relative error sets the reliability of our model, thus yielding the threshold of error within which an estimate can be considered good.

\section{REFERENCES}

[1] A. Bicchi, MA. Peshkin, and JE Colgate. Safety for physical humanrobot interaction. In B Siciliano and O. Khatib, editors, Springer Handbook of Robotics, pages 1335-1348. Springer, New York, 2008.

[2] A. Bicchi and G. Tonietti. Fast and soft arm tactics: Dealing with the safety-performance trade-off in robots arms design and control. IEEE Robotics and Automation Magazine, 11(2):22-33, 2004.

[3] J. Cho, S. Hong, W. Lee, and S. Kang. A variable stiffness joint using leaf springs for robot manipulators. In IEEE International Conference on Robotics and Automation, pages 4363-4368, 2009.

[4] J. Choi, S. Park, W. Lee, and S-C. Kang. Design of a robot joint with variable stiffness. In IEEE International Conference on Robotics and Automation, pages 1760-1765, 2008.

[5] A. De Luca and F. Flacco. Dynamic gravity cancellation in robots with flexible transmissions. In IEEE Conference on Decison and Control, pages 288-295, 2010.

[6] A. De Luca, F. Flacco, A. Bicchi, and R. Schiavi. Nonlinear decoupled motion-stiffness control and collision detection/reaction for the vsa-ii variable stiffness device. In IEEE/RSJ International Conference on Intelligent Robots and Systems, pages 5487-5494, 2009.

[7] Fabrizio Flacco, Alessandro De Luca, Irene Sardellitti, and Nikos G Tsagarakis. On-line estimation of variable stiffness in flexible robot joints. The International Journal of Robotics Research, 31(13):1556$1577,2012$.

[8] M. Garabini, A. Passaglia, F. Belo, P. Salaris, and A. Bicchi. Optimality principles in variable stiffness control: The vsa hammer. In IEEE/RSJ International conference on Intelligent Robots and Systems, pages 37703775,2011 


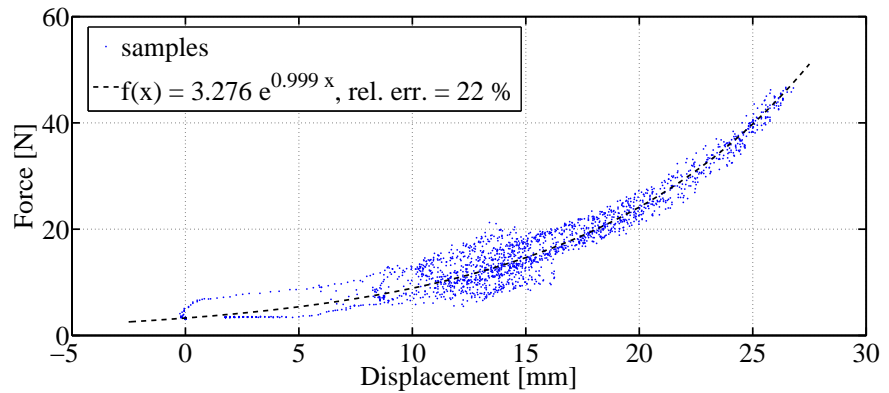

(a) left spring

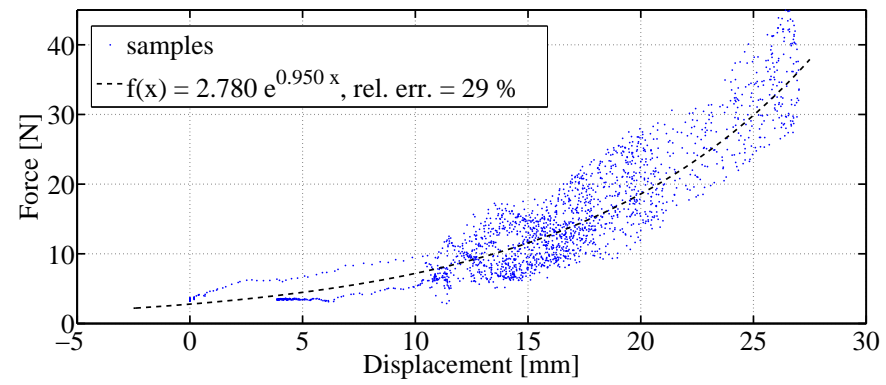

(b) right spring

Fig. 10. Experimental characterization of the two exponential springs. Force and displacement pairs recorded during a calibration experiment, and regression curve are shown for each of the two springs of the experimental VSA system.

[9] G. Grioli and A. Bicchi. A non-invasive real-time method for measuring variable stiffness. In Robotics science and systems, 2010.

[10] G. Grioli and A. Bicchi. A real-time parametric stiffness observer for vsa devices. In IEEE International Conference on Robotics and Automation, pages 5535-5540, 2011.

[11] S. Haddadin, T. Laue, U. Frese, S. Wolf, A. Albu-Schaeffer, and G. Hirzinger. Kick it like a safe robot: requirements for 2050. In Proceedings of Robotics and Autonomous systems, 2009.

[12] S. Haddadin, T. Laue, U. Frese, S. Wolf, A. Albu-Schäffer, and G. Hirzinger. Kick it with elasticity: safety and performance in human robot soccer. Robotics and Autonomous systems, 57:761-775, 2009.

[13] J.W. Hurst, J.E. Chestnutt, and A.A. Rizzi. An actuator with physically variable stiffness for highly dynamic legged locomotion. In IEEE International Conference on Robotics and Automation, pages 46624667, 2004.

[14] Y. Ikegami, K. Nagai, R.C.V. Loureiro, and W.S. Harwin. Design of redundant drive joint with adjustable stiffness and damping mechanism to improve joint admittance. In IEEE International Conference on Rehabilitation Robotics, pages 202-210, 2009.

[15] K. Ikuta, H. Ishii, and M. Nokata. Safety evaluation method of design and control for human-care robots. The international journal of robotics research, 22:281-297, 2003.

[16] A. Jafari, N.G. Tsagarakis, B. Vanderborght, and D.G. Cladwell. A novel actuator with adjustable stiffness (awas). In IEEE/RSJ International Conference on Intelligent Robots and Systems, pages 4201-4206, 2010.

[17] B.S. Kim and J.B. Song. Hybrid dual actuator unit: A design of a variable stiffness actuator based on an adjustable moment arm mechanism. In IEEE International Conference on Robotics and Automation, pages 1655-1660, 2010.

[18] D-Y Liu, O. Gibaru, and W. Perruquetti. Error analysis of a class of derivative estimators for noisy signals. Numerical algorithms, 58 (1):5383, 2011.

[19] L. Ljung. System identification. Springer, 1998.

[20] S. Migliore, E. Brown, and S. De Weerth. Biologically inspired joint stiffness control. In IEEE International Conference on Robotics and Automation, pages 4508-4513, 2005.

[21] T. Ménard, G. Grioli, and A. Bicchi. A real time observer for an agonistantagonist variable stiffness actuator. In IEEE International Conference on Robotics and Automation, pages 3988 - 3993, 2013.

[22] K.H. Nam, B.S. Kim, and J.B. Song. Compliant actuation of parallel- type variable stiffness actuator based on antagonistic actuation. Journal of mechanical science and technology, 24(11):2315-2321, 2010.

[23] G. Palli, C. Melchiorri, and A. De Luca. On the feedback linearization of robots with variable joint stiffness. In IEEE International Conference on Robotics and Automation, pages 1753-1759, 2008.

[24] G.A. Pratt and M.M. Williamson. Series elastic actuators. In IEEE/RSJ International Conference on Intelligent Robots and Systems, pages 399406, 1995.

[25] H.A. Preising and D.W.T. Rippin. Theory and application of the modulating function method i: Review and theory of the method of the splinetype modulating functions. Computers and Chemical Engineering, 17:116, 1993.

[26] I. Sardellitti, G. Medrano-Cerda, N. Tsagarakis, A. Jafaris, and D. Caldwell. A position and stiffness control strategy for variable stiffness actuators. In IEEE International Conference on Robotics and Automation, pages 2785-2791, 2012.

[27] R. Schiavi, G. Grioli, S. Sen, and A. Bicchi. Vsa-II: A novel prototype of variable stiffness actuator for safe and performing interacting with humans. In IEEE International Conference on Robotics and Automation, pages 2171-2176, 2008.

[28] M. Uemura and S. Kawamura. Resonance-based motion control method for multi-joint robot through combining stiffness adaptation and iterative learning control. In IEEE International Conference on Robotics and Automation, 2009.

[29] R. Van Ham, T. Sugar, B. Vanderborght, K. Hollander, and D. Lefeber. Review of actuators with passive adjustable compliance/controllable stiffness for robotic apllications. IEEE Robotics and Automation Magazine, 16(3):81-94, 2009.

[30] R. Van Ham, B. Vanderborght, M. Van Damme, B. Verrelst, and D. Lefeber. Maccepa, the mechanical adjustable compliance and controllable equilibrium position actuator: Design and implementation in a biped robot. Robotics and Autonomous Systems, 10:761-768, 2007.

[31] L. Visser, R. Carloni, and S. Stramigioli. Energy efficient variables stiffness actuators. IEEE Transactions on Robotics, 27:865-875, 2011.

[32] R.J. Wang and H.P. Huang. Avse - active variable stiffness exoskeleton robot system: Design and application for safe active-passive elbow rehabilitation. In IEEE/ASME International Conference on Advanced Intelligent Mechatronics, pages 220-225, 2012.

[33] T. Wimbock, C. Ott, A. Albu-Schaffer, A. Kugi, and G. Hirzinger. Impedance control for variable stiffness mechanisms with nonlinear joint coupling. In IEEE/RSJ International Conference on Intelligent Robots and Systems, pages 3796-3803, 2008. 